\title{
The graph based on Gröbner-Shirshov bases of groups
}

\author{
Eylem G Karpuz ${ }^{1 *}$, Firat Ates ${ }^{2}$, A Sinan Çevik ${ }^{3}$ and I Naci Cangul ${ }^{4}$
}

"Correspondence:

eylem.guzel@kmu.edu.tr

${ }^{1}$ Department of Mathematics, Kamil

Özdag Science Faculty,

Karamanoglu Mehmetbey

University, Yunus Emre Campus,

Karaman, 70100, Turkey

Full list of author information is

available at the end of the article

\begin{abstract}
Let us consider groups $G_{1}=\mathbb{Z}_{k} *\left(\mathbb{Z}_{m} * \mathbb{Z}_{n}\right), G_{2}=\mathbb{Z}_{k} \times\left(\mathbb{Z}_{m} * \mathbb{Z}_{n}\right), G_{3}=\mathbb{Z}_{k} *\left(\mathbb{Z}_{m} \times \mathbb{Z}_{n}\right)$, $G_{4}=\left(\mathbb{Z}_{k} * \mathbb{Z}_{l}\right) *\left(\mathbb{Z}_{m} * \mathbb{Z}_{n}\right)$ and $G_{5}=\left(\mathbb{Z}_{k} * \mathbb{Z}_{l}\right) \times\left(\mathbb{Z}_{m} * \mathbb{Z}_{n}\right)$, where $k_{1}, m_{1}, n \geq 2$. In this paper, by defining a new graph $\Gamma\left(G_{i}\right)$ based on the Gröbner-Shirshov bases over groups $G_{i}$, where $1 \leq i \leq 5$, we calculate the diameter, maximum and minimum degrees, girth, chromatic number, clique number, domination number, degree sequence and irregularity index of $\Gamma\left(G_{i}\right)$. Since graph theoretical studies (including such above graph parameters) consist of some fixed point techniques, they have been applied in such fields as chemistry (in the meaning of atoms, molecules, energy etc.) and engineering (in the meaning of signal processing etc.), game theory and physics. In addition, the Gröbner-Shirshov basis and the presentations of algebraic structures contain a mixture of algebra, topology and geometry within the purposes of this journal.

MSC: $05 C 25 ; 13 \mathrm{P} 10 ; 20 \mathrm{M} 05 ; 20 \mathrm{E} 06 ; 26 \mathrm{C} 10$
\end{abstract}

Keywords: graphs; Gröbner-Shirshov bases; group presentation

\section{Introduction and preliminaries}

In $[1,2]$, the authors have recently developed a new approach between algebra (in the meaning of groups and monoids) and analysis (in the meaning of generating functions). In a similar manner, in this paper, we make a connection between graph theory and GröbnerShirshov bases. In the literature, there are no works related to the idea of associating a graph with the Gröbner-Shirshov basis of a group. So, we believe that this paper will be the first work in that direction. As we depicted in the abstract of this paper, while graph theoretical studies actually consist of some fixed point techniques, so they have been applied in different branches of science such as chemistry (in the meaning of atoms, molecules, energy etc.) and engineering (in the meaning of signal processing etc.), Gröbner-Shirshov bases and algebraic presentations contain a mixture of algebra, topology and geometry within the purposes of this journal.

In detail, in this paper, we investigate the interplay between the group-theoretic property of a group $G$ and the graph-theoretic properties of $\Gamma(G)$ which is associated with $G$. By group-theoretic property, while we deal with the Gröbner-Shirshov basis of a given group, by graph-theoretic property, we are interested in the diameter, maximum and minimum degrees, girth, chromatic number, clique number, domination number, degree sequence and irregularity index of the corresponding graph of group. In the literature, there are some important graph varieties and works that are related to algebraic and topological

(c) 2013 G Karpuz et al.: licensee Springer. This is an Open Access article distributed under the terms of the Creative Commons Attribution License (http://creativecommons.org/licenses/by/2.0), which permits unrestricted use, distribution, and reproduction in any medium, provided the original work is properly cited. 
structures, namely, Cayley graphs [3-5] and zero-divisor graphs [6-8]. But the graph constructed in here is different from the previous studies and is also interesting in terms of using Gröbner-Shirshov basis theory during the construction of the vertex and edge sets. So, this kind of graph provides not only the classification of algebras (groups, semigroups), but also solving the problems of normal forms of elements, word problem, rewriting system, embedding theorems, extensions of groups and algebras, growth function, Hilbert series, etc. As is well known, these problems are really important in fixed point results since they have a direct connection to nature sciences.

Throughout this paper, for $k, l, m, n \geq 2$, we consider special groups $G_{1}=\mathbb{Z}_{k} *\left(\mathbb{Z}_{m} * \mathbb{Z}_{n}\right)$, $G_{2}=\mathbb{Z}_{k} \times\left(\mathbb{Z}_{m} * \mathbb{Z}_{n}\right), G_{3}=\mathbb{Z}_{k} *\left(\mathbb{Z}_{m} \times \mathbb{Z}_{n}\right), G_{4}=\left(\mathbb{Z}_{k} * \mathbb{Z}_{l}\right) *\left(\mathbb{Z}_{m} * \mathbb{Z}_{n}\right)$ and $G_{5}=\left(\mathbb{Z}_{k} * \mathbb{Z}_{l}\right) \times$ $\left(\mathbb{Z}_{m} * \mathbb{Z}_{n}\right)$ associated with the presentations

$$
\left.\begin{array}{l}
\mathcal{P}_{G_{1}}=\left\langle x, a, b ; x^{k}=1, a^{m}=1, b^{n}=1\right\rangle, \\
\mathcal{P}_{G_{2}}=\left\langle x, a, b ; x^{k}=1, a^{m}=1, b^{n}=1, x a=a x, x b=b x\right\rangle, \\
\mathcal{P}_{G_{3}}=\left\langle x, a, b ; x^{k}=1, a^{m}=1, b^{n}=1, a b=b a\right\rangle, \\
\mathcal{P}_{G_{4}}=\left\langle x, y, a, b ; x^{k}=1, y^{l}=1, a^{m}=1, b^{n}=1\right\rangle, \\
\mathcal{P}_{G_{5}}=\left\langle x, y, a, b ; x^{k}=1, y^{l}=1, a^{m}=1, b^{n}=1, x a=a x, x b=b x, y a=a y, y b=b y\right\rangle,
\end{array}\right\}
$$

respectively. By recalling the fundamentals of the Gröbner-Shirshov (GS) basis and then obtaining the GS-basis of each above group $G_{i}$, in Section 1.1, we define new simple, undirected graphs $\Gamma\left(G_{i}\right)$ associated with GS-bases of these groups. Then in Section 2, we compute the diameter, maximum and minimum degrees, girth, chromatic, clique and domination numbers, degree sequence and finally irregularity index of graphs $\Gamma\left(G_{i}\right)$ for each $1 \leq i \leq 5$.

Remark 1 The reason for us to present our results on these above parameters actually comes from their equality status. In other words, each result will be a good example for certain equalities over graph theoretical theorems.

(I) Preliminaries for graph theory. We first recall that for any simple graph $G$, the distance (length of the shortest path) between two vertices $u, v$ of $G$ is denoted by $d_{G}(u, v)$. If no such path exists, we set $d(x, y):=\infty$. Actually, the diameter of $G$ is defined by

$$
\operatorname{diam}(G)=\max \left\{d_{G}(x, y): x \text { and } y \text { are vertices of } G\right\} .
$$

The degree $\operatorname{deg}_{G}(v)$ of a vertex $v$ of $G$ is the number of vertices adjacent to $v$. Among all degrees, the maximum degree $\Delta(G)$ (or the minimum degree $\delta(G)$ ) of $G$ is the number of the largest (or the smallest) degrees in $G$ ([9]).

It is known that the girth of a simple graph $G, \operatorname{gr}(G)$ is the length of the shortest cycle contained in $G$. However, if $G$ does not contain any cycle, then the girth of it is assumed to be infinity.

Basically the coloring of $G$ is an assignment of colors (elements of some set) to the vertices of $G$, one color to each vertex, so that adjacent vertices are assigned distinct colors. If $n$ colors are used, then the coloring is referred to as $n$-coloring. If there exists an $n$-coloring of $G$, then $G$ is called $n$-colorable. The minimum number $n$ for which $G$ is $n$-colorable is called a chromatic number of $G$ and is denoted by $\chi(G)$. There exists another graph parameter, namely the clique of a graph $G$. In fact, depending on the vertices, each of the maximal 
complete subgraphs of $G$ is called a clique. Moreover, the largest number of vertices in any clique of $G$ is called the clique number and denoted by $\omega(G)$. In general, $\chi(G) \geq \omega(G)$ for any graph $G[9]$ and it is worth studying the cases that imply equality.

A subset $D$ of the vertex set $V(G)$ of a graph is called a dominating set if every vertex $V(G) \backslash D$ is joined to at least one vertex of $D$ by an edge. Additionally, the domination number $\gamma(G)$ is the number of vertices in the smallest dominating set for $G$ [9].

There also exists the term degree sequence, denoted by $\operatorname{DS}(G)$, which is a sequence of degrees of vertices of $G$. In [10], a new parameter for graphs has been recently defined, namely the irregularity index of $G$ denoted by $t(G)$, which is the number of distinct terms in the set DS(G).

(II) Preliminaries for the Gröbner-Shirshov basis. Since the main body of the paper is built up by considering the Gröbner-Shirshov (GS) basis, it is worth presenting some historical background about it as in the following.

The Gröbner basis theory for commutative algebras, which provides a solution to the reduction problem for commutative algebras, was introduced by Buchberger [11]. In [12], Bergman generalized the Gröbner basis theory to associative algebras by proving the 'diamond lemma.' On the other hand, the parallel theory of Gröbner bases was developed for Lie algebras by Shirshov [13]. The key ingredient of the theory is the so-called composition lemma which characterizes the leading terms of elements in the given ideal. In [14], Bokut noticed that Shirshov's method works for associative algebras as well. Thus, for this reason, Shirshov's theory for Lie algebras and their universal enveloping algebras is called the Gröbner-Shirshov basis theory. There are some specific studies on this subject related to some algebraic structures (see, for instance, [15-17]). We may finally refer to the papers [18-24] for some other recent studies of Gröbner-Shirshov bases. In the following, we give some fundamental facts about this important subject.

Let $K$ be a field, and let $K\langle X\rangle$ be the free associative algebra over $K$ generated by $X$. Denote by $X^{*}$ the free monoid generated by $X$, where the empty word is the identity denoted by 1 . For a word $w \in X^{*}$, we denote the length of $w$ by $|w|$. Suppose that $X^{*}$ is a well-ordered set. Then every nonzero polynomial $f \in K\langle X\rangle$ has the leading word $\bar{f}$. If the coefficient of $\bar{f}$ in $f$ is equal to one, then $f$ is called monic.

Let $f$ and $g$ be two monic polynomials in $K\langle X\rangle$. We then have two compositions as follows:

- If $w$ is a word such that $w=\bar{f} b=a \bar{g}$ for some $a, b \in X^{*}$ with $|\bar{f}|+|\bar{g}|>|w|$, then the polynomial $(f, g)_{w}=f b-a g$ is called the intersection composition of $f$ and $g$ with respect to $w$. The word $w$ is called an ambiguity of intersection.

- If $w=\bar{f}=a \bar{g} b$ for some $a, b \in X^{*}$, then the polynomial $(f, g)_{w}=f$ - agb is called the inclusion composition of $f$ and $g$ with respect to $w$. The word $w$ is called an ambiguity of inclusion.

If $g$ is monic, $\bar{f}=a \bar{g} b$ and $\alpha$ is the coefficient of the leading term $\bar{f}$, then transformation $f \mapsto f-\alpha a g b$ is called elimination (ELW) of the leading word of $g$ in $f$.

Let $S \subseteq K\langle X\rangle$ with each $s \in S$ be monic. Then the composition $(f, g)_{w}$ is called trivial modulo $(S, w)$ if $(f, g)_{w}=\sum \alpha_{i} a_{i} s_{i} b_{i}$, where each $\alpha_{i} \in K, a_{i}, b_{i} \in X^{*}, s_{i} \in S$ and $a_{i} \overline{s_{i}} b_{i}<w$. If this is the case, then we write $(f, g)_{w} \equiv 0 \bmod (S, w)$. We call the set $S$ endowed with the well-ordering < a Gröbner-Shirshov basis for $K\langle X \mid S\rangle$ if any composition $(f, g)_{w}$ of polynomials in $S$ is trivial modulo $S$ and corresponding $w$. 
The following lemma was proved by Shirshov [13] for free Lie algebras with deg-lex ordering (see also [25]). In 1976, Bokut [14] specialized Shirshov's approach to associative algebras (see also [12]). Meanwhile, for commutative polynomials, this lemma is known as Buchberger's theorem (see $[11,26]$ ).

Lemma 1 (Composition-diamond lemma) Let $K$ be a field,

$$
A=K\langle X \mid S\rangle=K\langle X\rangle / \operatorname{Id}(S)
$$

and let $<$ be a monomial ordering on $X^{*}$, where $\operatorname{Id}(S)$ is the ideal of $K\langle X\rangle$ generated by $S$. Then the following statements are equivalent:

$1 S$ is a Gröbner-Shirshov basis.

$2 f \in \operatorname{Id}(S) \Rightarrow \bar{f}=a \bar{s} b$ for some $s \in S$ and $a, b \in X^{*}$.

$3 \operatorname{Irr}(S)=\left\{u \in X^{*} \mid u \neq a \bar{s} b, s \in S, a, b \in X^{*}\right\}$ is a basis for the algebra $A=K\langle X \mid S\rangle$.

If a subset $S$ of $K\langle X\rangle$ is not a Gröbner-Shirshov basis, then we can add to $S$ all nontrivial compositions of polynomials of $S$, and by continuing this process (maybe infinitely), we eventually obtain a Gröbner-Shirshov basis $S^{\text {comp }}$. Such a process is called the Shirshov algorithm.

\subsection{A new graph based on GS-bases}

In the following, for $1 \leq i \leq 5$, by taking into account each group $G_{i}$ presented by $\mathcal{P}_{G_{i}}=$ $\left\langle X_{i} ; R_{i}\right\rangle$, as in (1), we define a undirected graph $\Gamma\left(G_{i}\right)=\left(V_{i}, E_{i}\right)$ and all results will be constructed on it.

The vertex $V_{i}$ and edge $E_{i}=\left\{\left(v_{p}, v_{q}\right)\right\}$ sets consist of

- generators of $G_{i}$,

- leading elements of polynomials in the GS basis of $G_{i}$,

- ambiguities of intersection or inclusion in the GS basis of $G_{i}$, and

- $v_{p}$ and $v_{q}$ form an ambiguity with each other,

- $\exists v_{r} \in X_{i}^{*}$ such that $v_{r}=x v_{p}$ or $v_{r}=v_{q} y$ for some $x, y \in X_{i}$,

- $v_{p}$ is reducible to $v_{q}$, respectively.

Since the Gröbner-Shirshov basis plays an important role in the definition of this new graph, let us define these bases for each of the groups $G_{i}$ where $1 \leq i \leq 5$. To do that, let us assume an ordering among the generators of $G_{i}(1 \leq i \leq 3)$ as $x>a>b$ and the generators of $G_{4}$ and $G_{5}$ as $x>y>a>b$.

Now, let us first consider $\mathcal{P}_{G_{1}}$. Since we have no intersection or inclusion compositions, the Gröbner-Shirshov basis of $G_{1}$ is $S_{1}=\left\{x^{k}-1, a^{m}-1, b^{n}-1\right\}$.

For $\mathcal{P}_{G_{2}}$, we have the ambiguities of intersection as $x^{k} a, x a^{m}, x^{k} b, x b^{n}$. Since these are trivial to modulo $R_{2}$, the Gröbner-Shirshov basis of $G_{2}$ is $S_{2}=\left\{x^{k}-1, a^{m}-1, b^{n}-1, x a-\right.$ $a x, x b-b x\}$.

For $\mathcal{P}_{G_{3}}$, we have the ambiguities of intersection as $a^{m} b$ and $a b^{n}$. Since these are trivial to modulo $R_{3}$, the Gröbner-Shirshov basis of $G_{3}$ is $S_{3}=\left\{x^{k}-1, a^{m}-1, b^{n}-1, a b-b a\right\}$.

For $\mathcal{P}_{G_{4}}$, since we have no intersection or inclusion compositions, the Gröbner-Shirshov basis of $G_{4}$ is $S_{4}=\left\{x^{k}-1, y^{l}-1, a^{m}-1, b^{n}-1\right\}$. 


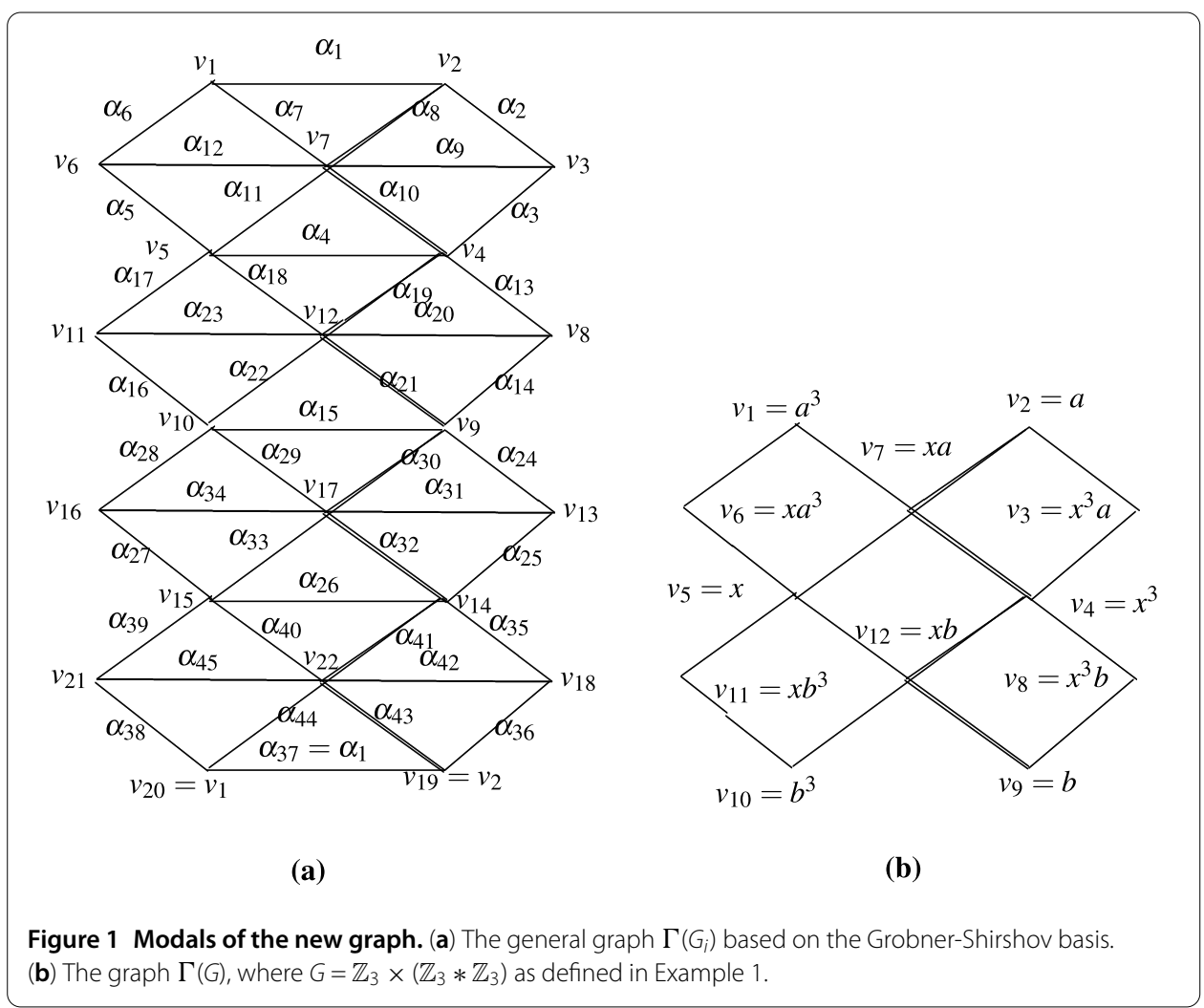

Finally, for $\mathcal{P}_{G_{5}}$, we have the ambiguities of intersection as

$$
x^{k} a, x a^{m}, x^{k} b, x b^{n}, y^{l} a, y a^{m}, y^{l} b, y b^{n} .
$$

Since these are trivial to modulo $R_{5}$, the Gröbner-Shirshov basis of $G_{5}$ is $S_{5}=\left\{x^{k}-1, y^{l}-\right.$ $\left.1, a^{m}-1, b^{n}-1, x a-a x, x b-b x, y a-a y, y b-b y\right\}$.

\section{Graph theoretical results over $\Gamma\left(G_{i}\right)$}

In this section, by considering the graph $\Gamma\left(G_{i}\right), 1 \leq i \leq 5$, we mainly deal with some graph properties, namely diameter, maximum and minimum degrees, girth, chromatic number, clique number, domination number, degree sequence and irregularity index of $\Gamma\left(G_{i}\right)$.

\subsection{Case 1: the graph $\Gamma\left(G_{1}\right)$, where $G_{1}=\mathbb{Z}_{k} *\left(\mathbb{Z}_{m} * \mathbb{Z}_{n}\right)$}

If we consider the graph of the group $G_{1}$, then we have a subgraph of Figure 1(a) with vertices $v_{1}=a^{m}, v_{2}=a, v_{4}=x^{k}, v_{5}=x, v_{9}=b$ and $v_{10}=b^{n}$. In this graph the edge set depends on the orders of factor groups of $G_{1}$. If we take $k, m, n=2$, then by the edge definition, we have the edges $\alpha_{1}, \alpha_{4}, \alpha_{15}$ in this subgraph of Figure 1(a). In the case $k, m, n>2$, we do not have any edges. In the remaining case, i.e., one or two orders of factor groups of $G_{1}$ are equal to two, we have one or two edges among $\alpha_{1}, \alpha_{4}$ and $\alpha_{15}$.

If we reconsider that the graph in Figure 1(a) depends on the group $G_{1}$ with above facts, then the picture will be shown as an unconnected graph which is not related to the numbers $k, m$ and $n$. Thus the first result is the following.

Theorem $1 \operatorname{diam}\left(\Gamma\left(G_{1}\right)\right)=\infty$. 
Theorem 2 The maximum and minimum degrees of the graph $\Gamma\left(G_{1}\right)$ are

$$
\Delta\left(\Gamma\left(G_{1}\right)\right)= \begin{cases}1 ; & \text { at least one of } k, m, n=2 \\ 0 ; & k, m, n>2\end{cases}
$$

and

$$
\delta\left(\Gamma\left(G_{1}\right)\right)= \begin{cases}1 ; & k, m, n=2 \\ 0 ; & \text { at most two of } k, m, n=2 \text { or } k, m, n>2\end{cases}
$$

respectively.

Proof If we take that at least one of $k, m, n$ is two, then we have at least one of the edges $\alpha_{1}, \alpha_{4}, \alpha_{15}$. Thus we get $\Delta\left(\Gamma\left(G_{1}\right)\right)=1$. If we consider $k=m=n=2$, then since we have edges $\alpha_{1}, \alpha_{4}, \alpha_{15}$, this gives us that all vertices in the graph $\Gamma\left(G_{1}\right)$ have degree one. So, $\Delta\left(\Gamma\left(G_{1}\right)\right)=\delta\left(\Gamma\left(G_{1}\right)\right)=1$. Now we consider the case $k, m, n>2$. In this case, since we have no edges in the graph $\Gamma\left(G_{1}\right)$, we obtain $\Delta\left(\Gamma\left(G_{1}\right)\right)=\delta\left(\Gamma\left(G_{1}\right)\right)=0$. If we take that at most two of $k, m, n$ are equal to two, then we get four vertices having degree one and two vertices having degree zero. Therefore, in this case, $\delta\left(\Gamma\left(G_{1}\right)\right)=0$.

Theorem 3 For any $k, m, n \neq 1$, the girth of the graph $\Gamma\left(G_{1}\right)$ is equal to infinity.

Proof Since we just have the edges $\alpha_{1}, \alpha_{4}$ and $\alpha_{15}$ depending on the numbers $k, m, n$, we do not have any cycle in the graph $\Gamma\left(G_{1}\right)$. So, $\operatorname{gr}\left(\Gamma\left(G_{1}\right)\right)=\infty$.

Theorem 4 The chromatic number of $\Gamma\left(G_{1}\right)$ is equal to

$$
\chi\left(\Gamma\left(G_{1}\right)\right)= \begin{cases}2 ; & \text { at least one of } k, m, n=2 \\ 1 ; & k, m, n>2\end{cases}
$$

Proof If we take that at least one of $k, m, n$ is two, then we have at least one of the edges $\alpha_{1}$, $\alpha_{4}, \alpha_{15}$. Thus we use two different colors since there exist neighbor vertices. By the edge definition of $\Gamma\left(G_{1}\right)$, we do not have any edges between generators and elements of three factor groups of $G_{1}$. Thus we obtain $\chi\left(\Gamma\left(G_{1}\right)\right)=2$. If we consider $k, m, n>2$, then since we do not have any edges in the graph, we can label all vertices with the same color. Therefore $\chi\left(\Gamma\left(G_{1}\right)\right)=1$.

Theorem 5 The clique number of $\Gamma\left(G_{1}\right)$ is equal to

$$
\omega\left(\Gamma\left(G_{1}\right)\right)= \begin{cases}2 ; & \text { at least one of } k, m, n=2, \\ 1 ; & k, m, n>2\end{cases}
$$

Proof The proof of this theorem is similar to the proof of Theorem 4 . If we take that at least one of $k, m, n$ is 2 , then we have at least one of the edges $\alpha_{1}, \alpha_{4}, \alpha_{15}$, i.e., we have a disconnected graph which has at least three complete subgraphs. Since these complete subgraphs have two vertices, we get $\omega\left(\Gamma\left(G_{1}\right)\right)=2$. If we consider $k, m, n>2$, then since we do not have any edges in the graph, the number of vertices in maximal clique is one. 
Theorem 6 The domination number of $\Gamma\left(G_{1}\right)$ is equal to infinity.

Proof For all cases of $k, m, n$, since the graph $\Gamma\left(G_{1}\right)$ is disconnected, we get $\gamma\left(\Gamma\left(G_{1}\right)\right)=$ $\infty$

Theorem 7 The degree sequence and irregularity index of $\Gamma\left(G_{1}\right)$ are given by

$$
\operatorname{DS}\left(\Gamma\left(G_{1}\right)\right)= \begin{cases}(1,1,1,1,1,1) ; & k, m, n=2, \\ (0,0,0,0,0,0) ; & k, m, n>2, \\ \left(i_{1}, i_{2}, i_{3}, i_{4}, i_{5}, i_{6}\right) ; & \text { otherwise }\end{cases}
$$

where $i_{j}=0,1$ for $1 \leq j \leq 6$, and

$$
t\left(\Gamma\left(G_{1}\right)\right)= \begin{cases}1 ; & k, m, n \geq 2, \\ 2 ; & \text { at least one of } k, m, n \text { is equal } 2,\end{cases}
$$

respectively.

Proof By the graph $\Gamma\left(G_{1}\right)$, if $k, m, n$ are equal to two and greater than two, then the degrees of the vertices are one and zero, respectively. But if at least one of $k, m, n$ is equal to two, then some vertices have degree one and some of them have degree zero. Hence, by the definition of a degree sequence, we clearly obtain the set $\operatorname{DS}\left(\Gamma\left(G_{1}\right)\right)$, as depicted. Nevertheless, it is easily seen that the irregularity index $t\left(\Gamma\left(G_{1}\right)\right)=1$ and 2 , as required.

\subsection{Case 2: the graph $\Gamma\left(G_{2}\right)$, where $G_{2}=\mathbb{Z}_{k} \times\left(\mathbb{Z}_{m} * \mathbb{Z}_{n}\right)$}

If we consider the graph of the group $G_{2}$, then we have a subgraph of Figure 1(a) with vertices $v_{1}=a^{m}, v_{2}=a, v_{3}=x^{k} a, v_{4}=x^{k}, v_{5}=x, v_{6}=x a^{m}, v_{7}=x a, v_{8}=x^{k} b, v_{9}=b, v_{10}=b^{n}$, $v_{11}=x b^{n}$ and $v_{12}=x b$. In this graph the edge set depends on the orders of factor groups of $G_{2}$. If we take $k, m, n=2$ then, by the edge definition, we have the edges $\alpha_{j}, 1 \leq j \leq 23$. In the case $k, m, n>2$, we do not have edges $\alpha_{1}, \alpha_{4}, \alpha_{9}, \alpha_{12}, \alpha_{15}, \alpha_{20}$ and $\alpha_{23}$ in $\Gamma\left(G_{2}\right)$.

Theorem 8 The diameter of the graph $\Gamma\left(G_{2}\right)$ is equal to four.

Proof By considering the graph of the group $G_{2}$, we say that the diameter of the graph $\Gamma\left(G_{2}\right)$ does not depend on the numbers $k, m, n$. For any $k, m, n$, in the graph $\Gamma\left(G_{2}\right)$ the vertices $v_{7}=x a$ and $v_{12}=x b$ are adjacent to vertices $v_{1}, v_{2}, v_{3}, v_{4}, v_{5}, v_{6}$ and $v_{4}, v_{5}, v_{8}, v_{9}$, $v_{10}, v_{11}$, respectively. If we connect any two vertices, except $v_{4}$ and $v_{5}$, via the shortest path, we need to pass through the vertices $v_{7}$ and $v_{12}$. For instance, we need the edges $\alpha_{7}, \alpha_{11}$, $\alpha_{18}$ and $\alpha_{22}$ to connect two vertices $v_{1}=a$ and $v_{10}=b$. This gives us $\operatorname{diam}\left(\Gamma\left(G_{2}\right)\right)=4$.

Theorem 9 The maximum and minimum degrees of the graph $\Gamma\left(G_{2}\right)$ are

$$
\Delta\left(\Gamma\left(G_{2}\right)\right)= \begin{cases}6 ; & k, m, n=2, \\ 4 ; & k, m, n>2\end{cases}
$$


and

$$
\delta\left(\Gamma\left(G_{2}\right)\right)= \begin{cases}3 ; & k, m, n=2 \\ 2 ; & k, m, n>2\end{cases}
$$

respectively.

Proof For $k, m, n=2$, in the graph of $G_{2}$ the vertices $v_{7}=x a$ and $v_{12}=x b$ are adjacent to vertices $v_{1}, v_{2}, v_{3}, v_{4}, v_{5}, v_{6}$ and $v_{4}, v_{5}, v_{8}, v_{9}, v_{10}, v_{11}$, respectively. Since these vertices have the largest degrees in $\Gamma\left(G_{2}\right)$, we get $\Delta\left(\Gamma\left(G_{2}\right)\right)=6$. The other vertices $v_{1}, v_{2}, v_{3}, v_{6}, v_{8}, v_{9}$, $v_{10}$ and $v_{12}$ have degree three and the remaining vertices $v_{4}$ and $v_{5}$ have degree five. So, the minimum degree of the graph $\Gamma\left(G_{2}\right)$ is $\delta\left(\Gamma\left(G_{2}\right)\right)=3$. Now we take $k, m, n>2$. In this case, we do not have edges $\alpha_{1}, \alpha_{4}, \alpha_{9}, \alpha_{12}, \alpha_{15}, \alpha_{20}$ and $\alpha_{23}$. Thus the vertices $v_{4}, \nu_{5}, \nu_{7}$, $v_{12}$ have degree four and the remaining vertices have degree two. So, $\Delta\left(\Gamma\left(G_{2}\right)\right)=4$ and $\delta\left(\Gamma\left(G_{2}\right)\right)=2$.

Theorem 10 The girth of the graph $\Gamma\left(G_{2}\right)$ is equal to

$$
\operatorname{gr}\left(\Gamma\left(G_{2}\right)\right)= \begin{cases}3 ; & k, m, n=2 \\ 4 ; & k, m, n>2\end{cases}
$$

Proof By the considering the graph of the group $G_{2}$, we have twelve triangles and five squares for $k=m=n=2$ and $k, m, n>2$, respectively. By the definition of girth, this gives us the required result.

Theorem 11 The chromatic number of the graph $\Gamma\left(G_{2}\right)$ is equal to

$$
\chi\left(\Gamma\left(G_{2}\right)\right)= \begin{cases}3 ; & \text { at least one of } k, m, n=2, \\ 2 ; & k, m, n>2 .\end{cases}
$$

Proof If the graph $\Gamma\left(G_{2}\right)$ has one of the following forms: $\mathbb{Z}_{2} \times\left(\mathbb{Z}_{m} * \mathbb{Z}_{n}\right), \mathbb{Z}_{k} \times\left(\mathbb{Z}_{2} * \mathbb{Z}_{n}\right)$, $\mathbb{Z}_{k} \times\left(\mathbb{Z}_{m} * \mathbb{Z}_{2}\right), \mathbb{Z}_{2} \times\left(\mathbb{Z}_{2} * \mathbb{Z}_{n}\right), \mathbb{Z}_{2} \times\left(\mathbb{Z}_{m} * \mathbb{Z}_{2}\right), \mathbb{Z}_{k} \times\left(\mathbb{Z}_{2} * \mathbb{Z}_{2}\right)$ or $\mathbb{Z}_{2} \times\left(\mathbb{Z}_{2} * \mathbb{Z}_{2}\right)$, then we have similar neighbors for the graphs of each group. So, we can label the vertices with three different colors. If $k, m, n \neq 2$, then in the graph of $G_{2}$ each vertex has two or four neighbors. In this graph, since the opposite vertices, which have an edge between them, can be labeled with the same color, we have two different colors. Hence $\chi\left(\Gamma\left(G_{2}\right)\right)=2$.

Theorem 12 The domination number of the graph $\Gamma\left(G_{2}\right)$ is

$$
\gamma\left(\Gamma\left(G_{2}\right)\right)= \begin{cases}2 ; & k, m, n=2 \\ 4 ; & k, m, n>2\end{cases}
$$

Proof Firstly, we consider the case $k=m=n=2$. Since the vertices $v_{7}=x a$ and $v_{12}=x b$ are connected with all other vertices in the graph of $G_{2}$, we can take the dominating set as $\left\{v_{7}, v_{12}\right\}$. Thus $\gamma\left(\Gamma\left(G_{2}\right)\right)=2$. In the case $k, m, n \neq 2$, since the number of edges is decreasing, the number of connected edges is decreasing as well. In this case, let us choose 
the dominating set as $\left\{v_{4}, v_{5}, v_{7}, v_{12}\right\}$. Every vertex, except the vertices in the dominating set, is joined to at least one vertex of this dominating set by an edge. Therefore we have $\gamma\left(\Gamma\left(G_{2}\right)\right)=4$.

Theorem 13 The clique number of $\Gamma\left(G_{2}\right)$ is equal to

$$
\omega\left(\Gamma\left(G_{2}\right)\right)= \begin{cases}3 ; & \text { at least one of } k, m, n=2, \\ 2 ; & k, m, n>2 .\end{cases}
$$

Proof In the graph $\Gamma\left(G_{2}\right)$, for $k=m=n=2$, we have twelve complete subgraphs. These are obtained by the vertices $v_{1}-v_{2}-v_{7}, v_{2}-v_{3}-v_{7}, v_{3}-v_{4}-v_{7}, v_{4}-v_{5}-v_{7}, v_{5}-v_{6}-v_{7}$, $v_{4}-v_{5}-v_{12}, v_{4}-v_{8}-v_{12}, v_{8}-v_{9}-v_{12}, v_{9}-v_{10}-v_{12}, v_{10}-v_{11}-v_{12}$ and $v_{5}-v_{11}-v_{12}$. Hence $\omega\left(\Gamma\left(G_{2}\right)\right)=3$. If $k, m, n \neq 2$, then we can find the smallest complete subgraphs as edges obtained by any two vertices in the graph $\Gamma\left(G_{2}\right)$. So, $\omega\left(\Gamma\left(G_{2}\right)\right)=2$.

Theorem 14 The degree sequence and irregularity index of $\Gamma\left(G_{2}\right)$ are given by

$$
\operatorname{DS}\left(\Gamma\left(G_{2}\right)\right)= \begin{cases}(3,3,3,5,5,3,6,3,3,3,3,6) ; & k, m, n=2, \\ (2,2,2,4,4,2,4,2,2,2,2,4) ; & k, m, n>2\end{cases}
$$

and

$$
t\left(\Gamma\left(G_{2}\right)\right)= \begin{cases}3 ; & k, m, n=2, \\ 2 ; & k, m, n>2\end{cases}
$$

respectively.

Proof It is easily seen by the graph $\Gamma\left(G_{2}\right)$.

Example 1 Let us consider the group $G=\mathbb{Z}_{3} \times\left(\mathbb{Z}_{3} * \mathbb{Z}_{3}\right)$ presented by

$$
\mathcal{P}_{G}=\left\langle x, a, b ; x^{3}=1, a^{3}=1, b^{3}=1, x a=a x, x b=b x\right\rangle,
$$

and $x>a>b$, the graph $\Gamma(G)$ as drawn in Figure 1(b), with the vertex set

$$
V(\Gamma(G))=\left\{x, a, b, x^{3}, a^{3}, b^{3}, x^{3} a, x^{3} b, x a^{3}, x b^{3}, x a, x b\right\} .
$$

By the result of Theorems 8 and 14, we have $\operatorname{diam}(\Gamma(G))=4, \Delta(\Gamma(G))=4, \delta(\Gamma(G))=2$, $\operatorname{gr}(\Gamma(G))=4, \chi(\Gamma(G))=2, \gamma(\Gamma(G))=4, \omega(\Gamma(G))=2, \operatorname{DS}(\Gamma(G))=(2,2,2,4,4,2,4,2,2,2$, $2,4)$ and $t(\Gamma(G))=2$.

\subsection{Case 3: the graph $\Gamma\left(G_{3}\right)$, where $G_{3}=\mathbb{Z}_{k} *\left(\mathbb{Z}_{m} \times \mathbb{Z}_{n}\right)$}

If we consider the graph of the group $G_{3}$, then we have a subgraph of Figure 1(a) with vertices $v_{1}=a^{m}, v_{2}=a, v_{3}=a b^{n}, v_{4}=b^{n}, v_{5}=b, v_{6}=a^{m} b, v_{7}=a b, v_{9}=x$ and $v_{10}=x^{k}$. If we take $k, m, n=2$, then by the edge definition, we have the edges $\alpha_{j}, 1 \leq j \leq 12$ and $\alpha_{15}$ in this subgraph of Figure 1(a). In the case $k, m, n>2$, we do not have edges $\alpha_{1}, \alpha_{4}, \alpha_{9}, \alpha_{12}, \alpha_{15}$ in the graph $\Gamma\left(G_{3}\right)$. 
Theorem 15 The maximum and minimum degrees of the graph $\Gamma\left(G_{3}\right)$ are

$$
\Delta\left(\Gamma\left(G_{3}\right)\right)= \begin{cases}6 ; & k, m, n=2, \\ 4 ; & k, m, n>2\end{cases}
$$

and

$$
\delta\left(\Gamma\left(G_{3}\right)\right)= \begin{cases}1 ; & k, m, n=2, \\ 0 ; & k, m, n>2\end{cases}
$$

respectively.

Proof Let us consider the graph $\Gamma\left(G_{3}\right)$ and take $k, m, n=2$. In this case, the vertex $v_{7}$ has the maximum degree six and the vertices $v_{9}$ and $v_{10}$ have the minimum degree one. But if we take $k, m, n>2$, then since there do not exist the edges $\alpha_{1}, \alpha_{4}, \alpha_{9}, \alpha_{12}$ and $\alpha_{15}$ in the graph $\Gamma\left(G_{3}\right)$, we obtain the maximum degree four by the vertex $v_{7}$ and the minimum degree zero by the vertices $v_{9}$ and $v_{10}$.

Theorem 16 The girth of the graph $\Gamma\left(G_{3}\right)$ is

$$
\operatorname{gr}\left(\Gamma\left(G_{3}\right)\right)= \begin{cases}3 ; & k, m, n=2, \\ 4 ; & k, m, n>2\end{cases}
$$

Proof Firstly, we take account of the case $k=m=n=2$. In this case, we have six triangles which have the edges $\alpha_{1}-\alpha_{7}-\alpha_{8}, \alpha_{2}-\alpha_{8}-\alpha_{9}, \alpha_{3}-\alpha_{9}-\alpha_{10}, \alpha_{4}-\alpha_{10}-\alpha_{11}, \alpha_{5}-\alpha_{11}-\alpha_{12}$, $\alpha_{6}-\alpha_{7}-\alpha_{12}$ in the graph $\Gamma\left(G_{3}\right)$. Thus $\operatorname{gr}\left(\Gamma\left(G_{3}\right)\right)=3$. Now we consider the case $k, m, n>2$. In this case, since we do not have the edges $\alpha_{1}, \alpha_{4}, \alpha_{9}, \alpha_{12}$ and $\alpha_{15}$, we have two squares which have the edges $\alpha_{1}-\alpha_{6}-\alpha_{5}-\alpha_{7}$ and $\alpha_{2}-\alpha_{3}-\alpha_{4}-\alpha_{7}$ in the graph $\Gamma\left(G_{3}\right)$. Therefore $\operatorname{gr}\left(\Gamma\left(G_{3}\right)\right)=4$.

Theorem 17 The chromatic number of the graph $\Gamma\left(G_{3}\right)$ is

$$
\chi\left(\Gamma\left(G_{3}\right)\right)= \begin{cases}3 ; & k, m, n=2, \\ 2 ; & k, m, n>2 .\end{cases}
$$

Proof Let us take $k=m=n=2$. In the graph $\Gamma\left(G_{3}\right)$, since the vertex $v_{7}$ is connected with all vertices except the vertices $v_{9}$ and $v_{10}$, this vertex must be labeled by a different color than other vertices. In addition, since other vertices are connected with each other doubly, they can be labeled by two different colors. This gives us $\chi\left(\Gamma\left(G_{3}\right)\right)=3$. In the case $k, m, n>$ 2 , since we have two squares, as in the previous proof, in the graph $\Gamma\left(G_{3}\right)$, it is enough to label two adjacent vertices by different colors. Hence $\chi\left(\Gamma\left(G_{3}\right)\right)=2$.

Theorem 18 The domination number of the graph $\Gamma\left(G_{3}\right)$ is equal to infinity.

Proof For all cases of $k, m, n$, since the graph $\Gamma\left(G_{3}\right)$ is disconnected, we get $\gamma\left(\Gamma\left(G_{1}\right)\right)=$ $\infty$ 
Theorem 19 The clique number of the graph $\Gamma\left(G_{3}\right)$ is equal to

$$
\omega\left(\Gamma\left(G_{3}\right)\right)= \begin{cases}3 ; & k, m, n=2, \\ 2 ; & k, m, n>2 .\end{cases}
$$

Proof For the case $k=m=n=2$, we have six maximal complete subgraphs of the graph $\Gamma\left(G_{3}\right)$ which are triangles as in the proof of Theorem 16 . Thus the largest number of the vertices in any maximal complete subgraph is three. If we take $k, m, n>2$, then we get eight maximal complete subgraphs, namely $\alpha_{2}, \alpha_{3}, \alpha_{5}, \alpha_{6}, \alpha_{7}, \alpha_{8}, \alpha_{10}$ and $\alpha_{11}$, having two vertices. So, $\omega\left(\Gamma\left(G_{3}\right)\right)=2$.

Theorem 20 The degree sequence and the irregularity index of $\Gamma\left(G_{3}\right)$ are given by

$$
\operatorname{DS}\left(\Gamma\left(G_{3}\right)\right)= \begin{cases}(3,3,3,3,3,3,6,1,1) ; & k, m, n=2, \\ (2,2,2,2,2,2,4,0,0) ; & k, m, n>2\end{cases}
$$

and

$$
t\left(\Gamma\left(G_{3}\right)\right)=3 ; \quad k, m, n \geq 2,
$$

respectively.

Proof It is easily seen by the graph of the group $G_{3}$.

\subsection{Case 4: the graph $\Gamma\left(G_{4}\right)$, where $G_{4}=\left(\mathbb{Z}_{k} * \mathbb{Z}_{l}\right) *\left(\mathbb{Z}_{m} * \mathbb{Z}_{n}\right)$}

If we consider the graph of the group $G_{4}$, then we get a subgraph $\Gamma\left(G_{4}\right)$ of the graph in Figure 1(a) with vertices $v_{1}=a^{m}, v_{2}=a, v_{4}=x^{k}, v_{5}=x, v_{9}=b, v_{10}=b^{n}, v_{14}=y^{l}$ and $v_{15}=y$. If we take $k, l, m, n=2$, then by Section 1.1, we obtain the edges $\alpha_{1}, \alpha_{4}, \alpha_{15}$ and $\alpha_{26}$ in $\Gamma\left(G_{4}\right)$. For the case $k, l, m, n>2$, we do not have any edges. On the other hand, since at most three orders of factor groups of $G_{4}$ are equal to two, we have at most three edges between $\alpha_{1}$, $\alpha_{4}, \alpha_{15}$ and $\alpha_{26}$.

Since the proof of each condition of the next result is quite similar to the related results over the group $G_{1}=\mathbb{Z}_{k} *\left(\mathbb{Z}_{m} * \mathbb{Z}_{n}\right)$ in Case 1, we omit it.

Theorem 21 Let us consider the group $G_{4}=\left(\mathbb{Z}_{k} * \mathbb{Z}_{l}\right) *\left(\mathbb{Z}_{m} * \mathbb{Z}_{n}\right)$ with its subgraph $\Gamma\left(G_{4}\right)$ as defined in the first paragraph of this case.

(i) The maximum and minimum degrees of the graph $\Gamma\left(G_{4}\right)$ are

$$
\Delta\left(\Gamma\left(G_{4}\right)\right)= \begin{cases}1 ; & \text { at least one of } k, m, n=2, \\ 0 ; & k, m, n>2\end{cases}
$$

and

$$
\delta\left(\Gamma\left(G_{4}\right)\right)= \begin{cases}1 ; & k, m, n=2, \\ 0 ; & k, m, n>2,\end{cases}
$$

respectively. 
(ii) For any $k, l, m, n$ different from one by considering a subgraph of Figure 1(a), the girth of the graph $\Gamma\left(G_{4}\right)$ is $\operatorname{gr}\left(\Gamma\left(G_{4}\right)\right)=\infty$.

(iii) The chromatic number of the graph $\Gamma\left(G_{4}\right)$ is equal to

$$
\chi\left(\Gamma\left(G_{4}\right)\right)= \begin{cases}2 ; & \text { at least one of } k, m, n=2, \\ 1 ; & k, m, n>2\end{cases}
$$

(iv) The clique number of $\Gamma\left(G_{4}\right)$ is equal to

$$
\omega\left(\Gamma\left(G_{4}\right)\right)= \begin{cases}2 ; & k, m, n=2 \\ 1 ; & k, m, n>2\end{cases}
$$

(v) The domination number of $\Gamma\left(G_{4}\right)$ is equal to infinity.

(vi) The degree sequence and the irregularity index of $\Gamma\left(G_{4}\right)$ are given by

$$
\operatorname{DS}\left(\Gamma\left(G_{4}\right)\right)= \begin{cases}(1,1,1,1,1,1,1,1) ; & k, m, n=2, \\ (0,0,0,0,0,0,0,0) ; & k, m, n>2, \\ \left(i_{1}, i_{2}, i_{3}, i_{4}, i_{5}, i_{6}, i_{7}, i_{8}\right) ; & \text { otherwise }\end{cases}
$$

where $i_{j}=0,1(1 \leq j \leq 8)$ and

$$
t\left(\Gamma\left(G_{4}\right)\right)= \begin{cases}1 ; & k, m, n \geq 2, \\ 2 ; & \text { otherwise }\end{cases}
$$

respectively.

\subsection{Case 5: the graph $\Gamma\left(G_{5}\right)$, where $G_{5}=\left(\mathbb{Z}_{k} * \mathbb{Z}_{l}\right) \times\left(\mathbb{Z}_{m} * \mathbb{Z}_{n}\right)$}

Similarly as in Case 4 , for the group $G_{5}$, we obtain a subgraph $\Gamma\left(G_{5}\right)$ of the graph in Figure 1(a) having vertices $v_{1}=a^{m}, v_{2}=a, v_{3}=x^{k} a, v_{4}=x^{k}, v_{5}=x, v_{6}=x a^{m}, v_{7}=x a, v_{8}=x^{k} b$, $v_{9}=b, v_{10}=b^{n}, v_{11}=x b^{n}, v_{12}=x b, v_{13}=y^{l} b, v_{14}=y^{l}, v_{15}=y, v_{16}=y b^{n}, v_{17}=y b, v_{18}=y^{l} a$, $v_{19}=v_{2}=a, v_{20}=v_{1}=a^{m}, v_{21}=y a^{m}$ and $v_{22}=y a$. In this graph, the edge set depends on the orders of factor groups of $G_{5}$. If we take $k, l, m, n=2$, then, by the adjacency definition in Section 1.1, we have the edges $\alpha_{j}, 1 \leq j \leq 45$ with $\alpha_{1}=\alpha_{37}$. For the case $k, l, m, n>2$, we do not have any edges $\alpha_{1}, \alpha_{4}, \alpha_{9}, \alpha_{12}, \alpha_{15}, \alpha_{20}, \alpha_{23}, \alpha_{26}, \alpha_{31}, \alpha_{34}, \alpha_{37}=\alpha_{1}, \alpha_{42}, \alpha_{45}$ in $\Gamma\left(G_{5}\right)$.

In the following result (Theorem 22 below), we again omit the proof of it as in Theorem 21 since it is quite similar to the related results over the group $G_{2}=\mathbb{Z}_{k} \times\left(\mathbb{Z}_{m} * \mathbb{Z}_{n}\right)$ in Case 2.

Theorem 22 Let us consider the group $G_{5}=\left(\mathbb{Z}_{k} * \mathbb{Z}_{l}\right) \times\left(\mathbb{Z}_{m} * \mathbb{Z}_{n}\right)$ with its related graph $\Gamma\left(G_{5}\right)$ as defined in the first paragraph of Case 5 .

(i) The maximum and minimum degrees of the graph $\Gamma\left(G_{5}\right)$ are equal to

$$
\Delta\left(\Gamma\left(G_{5}\right)\right)= \begin{cases}6 ; & k, m, n=2 \\ 4 ; & k, m, n>2\end{cases}
$$


and

$$
\delta\left(\Gamma\left(G_{5}\right)\right)= \begin{cases}3 ; & k, m, n=2 \\ 2 ; & k, m, n>2\end{cases}
$$

respectively.

(ii) The girth of the graph $\Gamma\left(G_{5}\right)$ is

$$
\operatorname{gr}\left(\Gamma\left(G_{5}\right)\right)= \begin{cases}3 ; & k, m, n=2 \\ 4 ; & k, m, n>2\end{cases}
$$

(iii) The chromatic number of the graph $\Gamma\left(G_{5}\right)$ is

$$
\chi\left(\Gamma\left(G_{5}\right)\right)= \begin{cases}3 ; & \text { at least one of } k, m, n=2, \\ 2 ; & k, m, n>2 .\end{cases}
$$

(iv) The domination number of the graph $\Gamma\left(G_{5}\right)$ is

$$
\gamma\left(\Gamma\left(G_{5}\right)\right)= \begin{cases}4 ; & k, m, n=2 \\ 6 ; & k, m, n>2 .\end{cases}
$$

(v) The clique number of the graph $\Gamma\left(G_{5}\right)$ is

$$
\omega\left(\Gamma\left(G_{5}\right)\right)= \begin{cases}3 ; & k, m, n=2, \\ 2 ; & k, m, n>2 .\end{cases}
$$

(vi) The degree sequence and the irregularity index of $\Gamma\left(G_{5}\right)$ are given by

$$
\operatorname{DS}\left(\Gamma\left(G_{5}\right)\right)= \begin{cases}(5,5,3,5,5,3,6,3,5,5,3,6,3,5,5,3,6,3,5,5,3,6) ; & k, m, n=2, \\ (4,4,2,4,4,2,4,2,4,4,2,4,2,4,4,2,4,2,4,4,2,4) ; & k, m, n>2\end{cases}
$$

and

$$
t\left(\Gamma\left(G_{5}\right)\right)= \begin{cases}3 ; & k, m, n=2, \\ 2 ; & k, m, n>2,\end{cases}
$$

respectively.

\section{Competing interests}

The authors declare that they have no competing interests.

\section{Authors' contributions}

All authors completed the paper together. All authors read and approved the final manuscript.

\section{Author details}

${ }^{1}$ Department of Mathematics, Kamil Özdag Science Faculty, Karamanoglu Mehmetbey University, Yunus Emre Campus, Karaman, 70100, Turkey. ${ }^{2}$ Department of Mathematics, Faculty of Arts and Science, Balikesir University, Campus, Balikesir, 10100, Turkey. ${ }^{3}$ Department of Mathematics, Faculty of Science, Selçuk University, Campus, Konya, 42075, Turkey.

${ }^{4}$ Department of Mathematics, Faculty of Arts and Science, Uludag University, Gorukle Campus, Bursa, 16059, Turkey. 


\section{Acknowledgements}

Dedicated to Professor Hari M Srivastava.

All authors are partially supported by Research Project Offices (BAP) of their universities in Turkey.

Received: 26 January 2013 Accepted: 6 March 2013 Published: 26 March 2013

\section{References}

1. Cangul, IN, Cevik, AS, Simsek, Y: A new approach to connect algebra with analysis: relationships and applications between presentations and generating functions. Bound. Value Probl. 2013, 51 (2013). doi:10.1186/1687-2770-2013-51

2. Cevik, AS, Cangul, IN, Simsek, Y: An analysis approach to an extension of finite monoids. Fixed Point Theory Appl. 2013, 15 (2013). doi:10.1186/1687-1812-2013-15

3. Kelarev, AV, Praeger, CE: On transitive Cayley graphs of groups and semigroups. Eur. J. Comb. 24, 59-72 (2003)

4. Kelarev, AV: On Cayley graphs of inverse semigroups. Semigroup Forum 72, 411-418 (2006)

5. Luo, Y, Hao, Y, Clarke, GT: On the Cayley graphs of completely simple semigroups. Semigroup Forum 82, 288-295 (2011)

6. Anderson, DF, Livingston, PS: The zero-divisor graph of commutative ring. J. Algebra 217, 434-447 (1999)

7. Anderson, DF, Badawi, A: The zero-divisor graph of a ring. Commun. Algebra 36(8), 3073-3092 (2008)

8. DeMeyer, FR, DeMeyer, L: Zero-divisor graphs of semigroups. J. Algebra 283, 190-198 (2005)

9. Gross, JL: Handbook of Graph Theory. Chapman \& Hall/CRC Press, Boca Raton (2004)

10. Mukwembi, S: A note on diameter and the degree sequence of a graph. Appl. Math. Lett. 25, 175-178 (2012)

11. Buchberger, B: An Algorithm for Finding a Basis for the Residue Class Ring of a Zero-Dimensional Ideal. Ph.D. thesis, University of Innsbruck (1965)

12. Bergman, GM: The diamond lemma for ring theory. Adv. Math. 29, 178-218 (1978)

13. Shirshov, Al: Some algorithmic problems for Lie algebras. Sib. Math. J. 3, 292-296 (1962)

14. Bokut, LA: Imbedding into simple associative algebras. Algebra Log. 15, 117-142 (1976)

15. Bokut, LA, Vesnin, A: Gröbner-Shirshov bases for some Braid groups. J. Symb. Comput. 41, 357-371 (2006)

16. Chen, Y: Gröbner-Shirshov bases for Schreier extensions of groups. Commun. Algebra 36, 1609-1625 (2008)

17. Chen, Y, Zhong, C: Gröbner-Shirshov bases for HNN-extensions of groups and for the alternating group. Commun. Algebra 36, 94-103 (2008)

18. Ates, F, Karpuz, EG, Kocapinar, C, Cevik, AS: Gröbner-Shirshov bases of some monoids. Discrete Math. 311, 1064-1071 (2011)

19. Bokut, LA: Gröbner-Shirshov basis for the Braid group in the Birman-Ko-Lee generators. J. Algebra 321, 361-376 (2009)

20. Bokut, LA: Gröbner-Shirshov basis for the Braid group in the Artin-Garside generators. J. Symb. Comput. 43, 397-405 (2008)

21. Bokut, LA, Chen, Y, Zhao, X: Gröbner-Shirshov bases for free inverse semigroups. Int. J. Algebra Comput. 19(2), 129-143 (2009)

22. Bokut, LA, Chainikov, W: Gröbner-Shirshov basis of the Adyan extension of the Novikov group. Discrete Math. 308, 4916-4930 (2008)

23. Karpuz, EG, Cevik, AS: Gröbner-Shirshov bases for extended modular, extended Hecke and Picard groups. Math. Notes 92(5), 636-642 (2012)

24. Kocapinar, C, Karpuz, EG, Ates, F, Cevik, AS: Gröbner-Shirshov bases of the generalized Bruck-Reilly $*$-extension. Algebra Colloq. 19(1), 813-820 (2012)

25. Bokut, LA: Unsolvability of the word problem, and subalgebras of finitely presented Lie algebras. Izv. Akad. Nauk SSSR, Ser. Mat. 36, 1173-1219 (1972)

26. Buchberger, B: An algorithmical criteria for the solvability of algebraic systems of equations. Aequ. Math. 4, 374-383 (1970) (in German)

doi:10.1186/1687-1812-2013-71

Cite this article as: $\mathrm{G}$ Karpuz et al.: The graph based on Gröbner-Shirshov bases of groups. Fixed Point Theory and Applications 2013 2013:71 